\title{
Isolated Recurrence of Secondary CNS Lymphoma: Case Report and Literature Review
}

\author{
Peter DeRosa ${ }^{1}$ Justin M. Cappuzzo ${ }^{2}$ Jonathan H. Sherman ${ }^{1}$ \\ ${ }^{1}$ Department of Neurological Surgery, The George Washington \\ University, Washington, District of Columbia, United States \\ ${ }^{2}$ School of Medical and Health Sciences, The George Washington \\ University, Washington, District of Columbia, United States

\begin{abstract}
Address for correspondence Jonathan H. Sherman, MD, 2150 Pennsylvania Avenue, NW, Suite 7-420, Washington, DC, 20037, United States (e-mail: jsherman@mfa.gwu.edu).
\end{abstract} \\ J Neurol Surg Rep 2014;75:e154-e159.
}
Abstract
Keywords
- secondary central nervous system lymphoma
- isolated
- relapse
- recurrence
- aggressive treatment regimen

Isolated secondary central nervous system lymphoma (SCNSL) relapse is a rare disease. Consequently, standardized treatment regimens have yet to be developed. We present an interesting case of isolated SCNSL presenting with altered mental status and panhypopituitarism in a patient at low risk of developing the disease. We also review the related literature and discuss newer, more aggressive treatments for primary CNS lymphoma and SCNSL.

\section{Introduction}

Central nervous system (CNS) lymphoma can present as either secondary, representing 1 to $7 \%$ of lymphomas, or primary, representing 0.2 to $2 \%$ of lymphomas. ${ }^{1}$ Secondary CNS lymphoma (SCNSL)is currently defined as lymphoma not originating from within the CNS, and may be an isolated recurrence within the CNS or may be part of the systemic progressive disease. ${ }^{2}$ Additionally, SCNSL may be further categorized as leptomeningeal, parenchymal, or as a combination of the two. ${ }^{3}$ In particular, an isolated relapse within the CNS is rare, with retrospective cohorts typically numbering between 10 and 30 patients. ${ }^{4-7}$ Furthermore, due to a lack of clinical data on isolated SCNSL recurrence, features of the disease, a standard treatment regiment, and overall prognosis have yet to be elucidated. ${ }^{4,8}$

\section{Current Treatment of Primary and Secondary Central Nervous System Lymphoma}

Once the diagnosis has been established, treatment options for CNS lymphoma can be broadly divided into chemotherapy, radiation, and surgery. Surgery in CNS lymphoma has been reserved for diagnostic biopsy. Surgical resection of CNS lymphoma is generally a last resort and only offered in cases when the lesion is causing extreme mass effect and herniation syndromes. The mainstays of primary and secondary CNS lymphoma treatment are chemotherapy and radiation. Unfortunately, the standard chemotherapy regimens used in the treatment of systemic lymphomas have shown only little effect in prophylaxis or direct treatment of CNS lymphomas. ${ }^{9}$ These drugs (anthracyclines, vinca alkaloids, and some alkylating agents) have poor blood brain penetration, and their toxicity profiles limit the dose at which they can be delivered to overcome this. Methotrexate and cytarabine, in contrast, have poor blood brain barrier penetration but can be delivered at sufficient concentrations to overcome this and provide adequate concentration to the CNS. ${ }^{10}$ Initial studies showed improved overall survival from treatment with high-dose methotrexate and radiation at time of diagnosis, with response rates of up to 80 to $90 \%$ and 5-year median survival times in primary CNS lymphoma. ${ }^{11}$ Subsequent studies comparing high-dose methotrexate alone and combination high-dose methotrexate and radiation failed to prove robust survival benefit with combination therapy, especially in patients $>60$ years of age, a growing segment of the disease. ${ }^{11-13}$ received

September 10, 2014 accepted after revision April 4, 2014 published online June 4, 2014
DOI http://dx.doi.org/ 10.1055/s-0034-1378152. ISSN 2193-6358.
Copyright (c) 2014 by Georg Thieme Verlag KG, Inc., Stuttgart - New York Tel: +1(212) 584-4662.
License terms

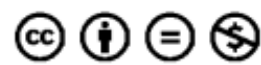


In addition, responses to radiation treatment have been found to be short lived and have failed to increase overall survival. ${ }^{14}$ The role of radiation therapy for CNS lymphoma has shifted from initial treatment to salvage therapy over the past several decades. ${ }^{9,15}$ Treatment paradigms established for primary CNS lymphoma have been used in the treatment of SCNSL; however, the failure rate remains high, with overall survival typically on the order of 2 months. ${ }^{16}$

More recently, more aggressive regimens have been suggested. These include surgical resection that has been shown to provide survival benefit in a single trial within a subset of patients who had a single CNS lesion in a noneloquent, surgically accessible region. ${ }^{17}$ Although there is no standard dosing for methotrexate, CNS concentration and response rates have been shown to be related to infusion rate. ${ }^{18}$ More specifically, methotrexate area under the curve has been shown to be an independent predictor of clinical outcome. ${ }^{19}$ Personalized dosing based on age, gender, and creatinine clearance has been proposed..$^{10}$ Blood brain barrier disruption methods in combination with chemotherapeutic agents that have been used successfully in systemic lymphomas has shown promise in early pilot studies and are currently being tested in larger centers. ${ }^{20,21}$ Similarly, intrathecal administration of methotrexate, cytarabine, or rituximab has shown some early promise, but further data are still required. Administration of intrathecal chemotherapy is generally reserved for patients with leptomeningeal disease and positivity on cerebrospinal fluid (CSF) testing, although large retrospective series have been equivocal on this topic. ${ }^{22,23}$

\section{Illustrative Case}

A 36-year-old man presented to his primary care physician with complaint of a soft nontender mass on the left side of his neck. The patient reported that he noticed this mass enlarging for the past year but had attributed it to an infectious process. An initial chest X-ray identified a left supraclavicular mass compressing the trachea and causing a rightward deviation. Additionally, a computed tomography (CT) scan of the chest and neck, with and without contrast, was performed for follow-up, with evidence of diffuse lymphadenopathy (-Fig. 1A), specifically, the supraclavicular, paratracheal, prevascular, lesser sac, left axilla, parasternal, and mesenteric chain nodes. A head CT scan with contrast performed at the same time was negative for any intracranial involvement. The patient also tested negative for human immunodeficiency virus.

The patient was subsequently scheduled for a CT-guided biopsy of the supraclavicular lesion. The results of the biopsy were consistent with B cell lymphoma. Following the biopsy confirmation, the patient completed six cycles of outpatient rituximab, cyclophosphamide, hydroxydaunorubicin, Oncovin, and prednisone therapy. At his last outpatient oncology visit, the patient was considered to be in remission. The patient did not experience any cognitive symptoms, and he had no extranodal involvement at diagnosis or follow-up. The patient's lactate dehydrogenase level was not measured as a part of his follow-up. This would place the patient at a low risk for developing a secondary CNS lymphoma relapse. 8,24

Approximately 7 months posttreatment, the patient was hospitalized at an outside medical center following a 1-week period of worsening mental status. On admission, the patient was found to have elevated serum sodium levels in the 160s, and further work-up revealed a suprasellar mass on head CT (-Fig. 2A). Upon arrival to our institution, the patient was found to have diabetes insipidus. With a known history of systemic lymphoma, the patient underwent a lumbar puncture for flow cytometry. The differential diagnoses also included germinoma, craniopharyngioma, and astrocytoma. A skull X-ray showed no calcifications. CT scan of the chest, abdomen, and pelvis displayed marked interval improvement in the degree of lymphadenopathy with near-complete resolution of the previous mediastinal lymphadenopathy (-Fig. 1B). CSF flow cytometry was negative for tumor cells. A brain magnetic resonance imaging (MRI) revealed a homogeneously enhancing suprasellar mass with surrounding edema (-Fig. 3A, B).

The patient was taken for a right fronto-orbital craniotomy and translaminar terminalis approach for biopsy of the lesion (-Fig. 2B). Pathology was consistent with B cell lymphoma. Histologically, the samples showed highly cellular clumps of lymphocytes on hematoxylin and eosin staining (-Fig. 4A-C), and immunohistochemical studies were positive for $\mathrm{Bcl} 2$, Bcl6, CD10, CD20, and CD79a (- Fig. 5A-E). Additionally, Ki67 staining ( $\mathbf{- F i g . ~ 5 F ) ~ h i g h l i g h t e d ~ t h e ~ p r o l i f e r a t i o n ~ o f ~} \sim 5$ to $10 \%$ of the neoplastic cells present in the biopsy. The patient was placed on high-dose methotrexate, and his mental status improved. Unfortunately, the patient's condition progressed and he succumbed to his illness.
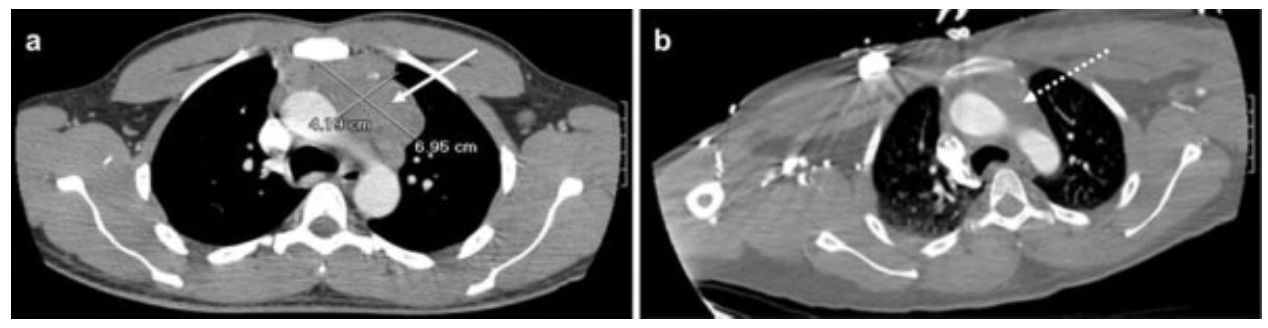

Fig. 1 (A) Pretreatment axial chest computed tomography (CT) with contrast revealing a prevascular lymph node mass (solid arrow) during the initial diagnosis of B-cell lymphoma. (B) Postchemotherapy axial chest CT with contrast illustrating near-complete resolution of the previously seen prevascular lymph node mass (dotted arrow). 

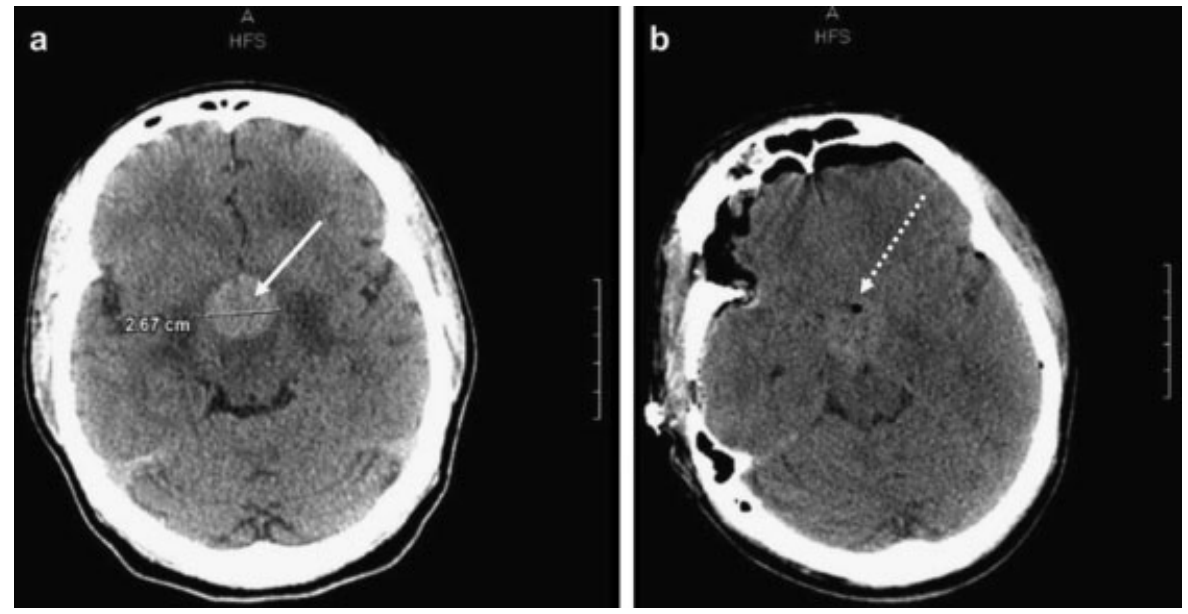

Fig. 2 (A) Preoperative axial head computed tomography (CT) without contrast from an outside institution revealing a 2.6-cm suprasellar mass (solid arrow). (B) Postoperative axial head CT without contrast illustrating the results of the right frontal orbital craniotomy for debulking and biopsy (dotted arrow).

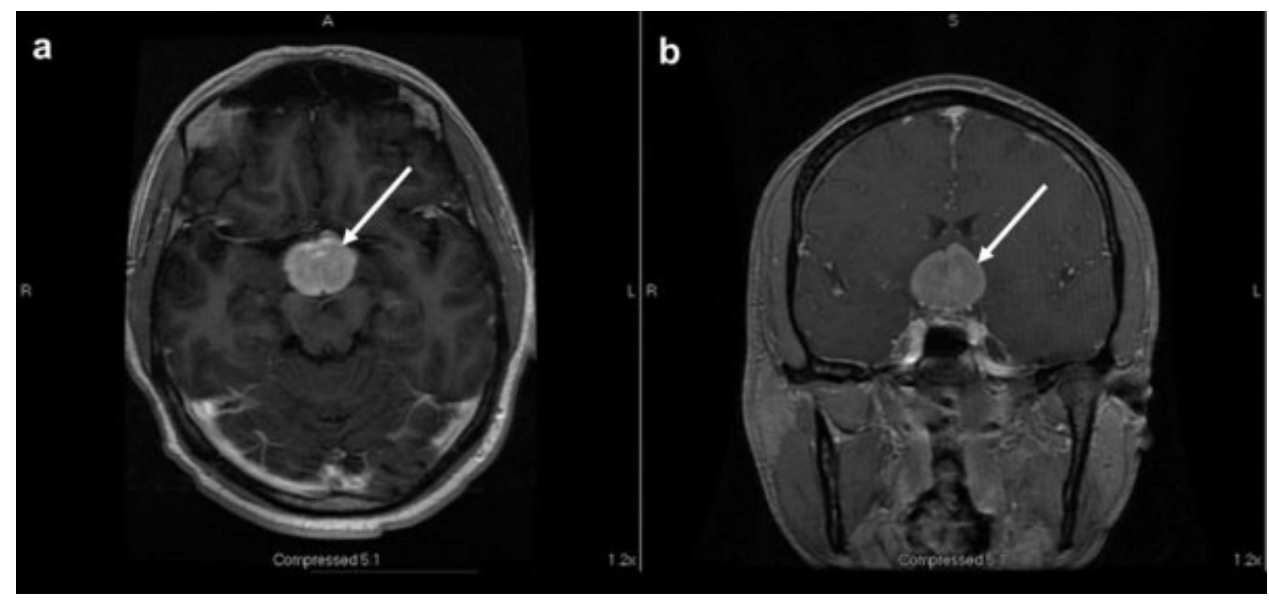

Fig. 3 Preoperative (A) axial and (B) coronal T1-weighted cranial magnetic resonance imaging with contrast, revealing a 3-cm suprasellar mass with surrounding edema (solid arrows).

\section{Discussion}

The incidence of SCNSL in the general population ranges from 4 to $23 \% .^{2,25-27}$ The factors that most significantly influence the incidence rate of SCNSL include the variant of primary lymphoma, involvement of more than one extranodal site, a serum lactate dehydrogenase level greater than three times the normal limit, an advanced stage of the systemic disease, and a high International Prognostic Index. .,3,28,29 $^{\text {Other }}$ studies have found that no one indicator of SCNSL is reliable on its own, but that the combination of several factors can help elucidate the risk of developing SCNSL and the need for possible prophylaxis. In particular, initial involvement of the breast, testis, and bone marrow with primary disease are heavily associated with an increased risk of developing SCNSL. ${ }^{30}$ Populations with the highest risk are those with immune deficiencies, either innate or acquired. In the 1980s, a steep increase in the incidence of SCNSL paralleled the increase in incidence of human immunodeficiency virus and autoimmune deficiency syndrome. However, with the advent of highly active antiretroviral therapy in the mid-1990s, the incidence has decreased and remained fairly steady since that time. The age of diagnosis of both primary and secondary CNS lymphoma has been steadily increasing, focusing more studies on treatment of an elderly population. ${ }^{31-35}$

As previously mentioned, the histologic grade of the primary lymphoma differentially influences the risk of developing SCNSL. Depending on whether the primary lymphoma is classified as indolent, aggressive, or highly aggressive, there is a $3 \%, 9 \%$, and $27 \%$ risk, respectively, of developing SCNSL. ${ }^{36}$ With specific regard to diffuse large B-cell lymphoma (DLBCL), the incidence has been reported as $5 \%$, but interestingly, when the primary lymphoma is of the mediastinal large B-cell variant, the risk of SCNSL climbs to $19 \%$, as in this case. Although most SCNLs present with leptomeningeal disease, DLBCL most often presents with parenchymal disease. ${ }^{3}$ Indolent lymphomas usually carry a low risk of recurrence, but when CNS disease has been found, it is usually after the histologic transformation to a more aggressive variant. ${ }^{27,37,38}$ Furthermore, presence of at least two of these: bone marrow, 

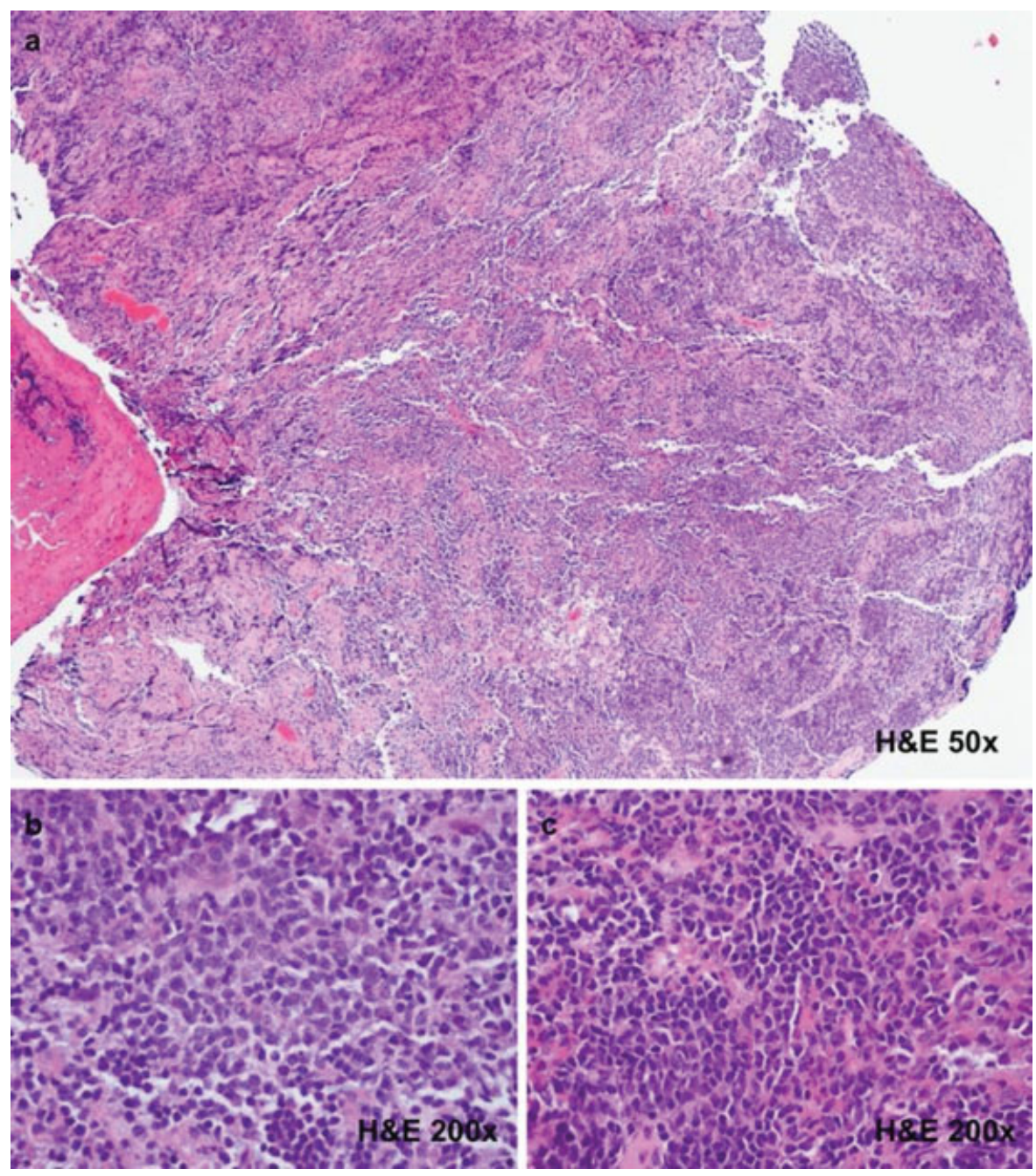

Fig. 4 Histopathology of the biopsied suprasellar mass under $(A)$ low magnification $(\times 50)$ and $(B, C)$ high magnification $(\times 200)$ revealing highly cellular clumps of lymphocytes consistent with diffuse large B-cell lymphoma (hematoxylin and eosin).

skin involvement, and B symptoms, increases the risk of SCNL in indolent primary lymphomas to $7 \% .8,24,28$

The characteristic clinical presentation of SCNSL is a newonset headache (50\%), palsies of cranial nerves III, IV, VI, and VII, changes in mental status (29\%), and even coma and seizures (23-29\%). ${ }^{39}$ It typically presents within 6 months of diagnosis of the primary lymphoma, which is typically confirmed with CSF studies and imaging. ${ }^{25,26}$ Flow cytometry, however, is more sensitive than CSF cytology, and polymerase chain reaction studies can be used for further confirmation. ${ }^{37,40}$ MRI is the current gold standard for localizing the recurrence because it has superior sensitivity compared with CT. Parenchymal lesions usually present with homogeneously enhancing superficial or periventricular lesions, but ring enhancement patterns can also be seen, especially in the population with acquired immunodeficiency syndrome.

SCNSL is characterized as an isolated recurrence $\sim 50 \%$ of the time; although most of these patients go on to develop systemic recurrences within several months. Isolated CNS recurrence has a worse prognosis than CNS disease at the time of diagnosis, suggesting an alternative disease mecha- nism. ${ }^{41}$ Survival is slightly better in isolated CNS recurrence when compared with systemic recurrence, which is the major cause of death in secondary CNS lymphoma. Unfortunately, the median survival time is $<6$ months when no treatment has been administered.

Treatment and CNS prophylaxis after the discovery of primary lymphoma is an area of active investigation. Based on the results of the large retrospective RICOVER-60 trial, intravenous (IV) rituximab is added to cyclophosphamide, doxorubicin, vincristine, and prednisolone (CHOP) therapy because it has shown a decreased incidence of SCNSL from 6.9 to $4.1 \%{ }^{24-26,39-43}$ Another study found that a regimen of ACVBP with IV methotrexate (MTX) may also be superior to standard CHOP therapy. ${ }^{30}$ The use of IV MTX has been shown to increase survival times for isolated SCNSL, especially for parenchymal SCNSL. ${ }^{44}$ Despite these results, a strong consensus has yet to be reached on the indications for, efficacy of, and means of CNS prophylaxis. This is partially due to the retrospective nature of the studies to date and the variance in treatment regimens among the different studies. Also, the histopathology of the primary lymphoma is a determining 

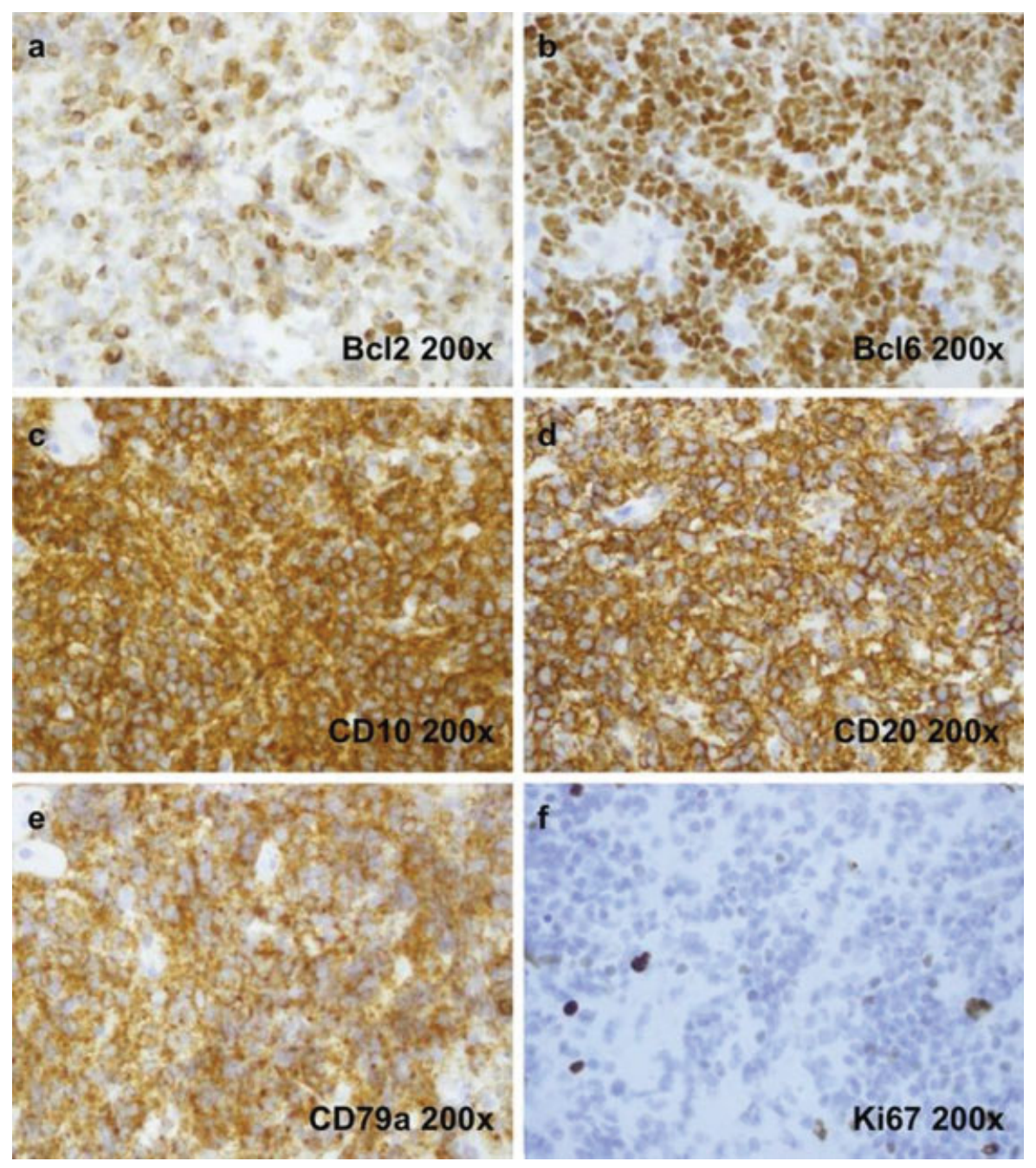

Fig. 5 Immunohistochemistry of the biopsied suprasellar mass stained positive for (A) Bcl2, (B) Bcl6, (C) CD10, (D) CD20, (E) CD79a, and (F) Ki-67, all consistent with diffuse large B-cell lymphoma $(\times 200)$.

factor in the efficacy of treatment. For example, although IV rituximab decreased the incidence of SCNSL overall, it does not seem to influence the incidence of SCNSL in DLBCL (30\%).

Interestingly, the patient in this case did not meet the criteria for increased risk of developing SCNSL, yet he still developed the disease. This leads us to believe that indeed no one factor is a definitive indicator that a patient will progress to a SCNSL. The classification of mediastinal DLBCL may have elevated the patient's risk of progression to SCNSL; however, his lack of other indicative risk factors made this an uncommon occurrence in an already uncommon condition. Despite the unusual presentation in this case (panhypopituitarism), the patient did have an isolated CNS relapse within 6 months, and the mental status change resolved in response to MTX treatment.

\section{Conclusions}

Isolated SCNSL is a rare disease in which standardized treatment guidelines have yet to be developed. This case is one particular example where a patient designated as low risk for developing SCNSL did progress to an isolated occurrence of the disease. Additionally, although treatment extended the patient's survival time and resolved the change in mental status, the disease continued to progress and the patient succumbed to his illness.

\section{Conflict of Interest}

The author has nothing to disclose.

\section{References}

1 Jellinger K, Radiaszkiewicz T. Involvement of the central nervous system in malignant lymphomas. Virchows Arch A Pathol Anat Histol 1976;370(4):345-362

2 Tomita N, Kodama F, Kanamori H, Motomura S, Ishigatsubo Y. Secondary central nervous system lymphoma. Int J Hematol 2006; 84(2):128-135

3 Feugier P, Virion JM, Tilly H, et al. Incidence and risk factors for central nervous system occurrence in elderly patients with diffuse large-B-cell lymphoma: influence of rituximab. Ann Oncol 2004; 15(1):129-133

4 Kawano N, Ochiai H, Yoshida S, et al. Clinical features and treatment outcomes of isolated secondary central nervous system lymphomas in Miyazaki Prefecture. Int J Clin Oncol 2012;17(4):336-340 
5 Kim UR, Shah AD, Arora V, Solanki U. Isolated optic nerve infiltration in systemic lymphoma-a case report and review of literature. Ophthal Plast Reconstr Surg 2010;26(4):291-293

6 Patrij K, Reiser M, Wätzel L, et al; International Primary CNS Lymphoma Collaborative Group (IPCG).Isolated central nervous system relapse of systemic lymphoma (SCNSL): clinical features and outcome of a retrospective analysis. Ger Med Sci 2011;9:Doc11

7 Stuplich M, Mayer K, Kim Y, et al. Richter syndrome and brain involvement: low-grade lymphoma relapsing as cerebral highgrade lymphoma. Acta Haematol 2012;127(2):93-95

8 van Besien K, Gisselbrecht C, Pfreundschuh M, Zucca E. Secondary lymphomas of the central nervous system: risk, prophylaxis and treatment. Leuk Lymphoma 2008;49(Suppl 1):52-58

9 Roth P, Stupp R, Eisele G, Weller M. Treatment of primary CNS lymphoma. Curr Treat Options Neurol 2014;16(1):277

10 Prodduturi P, Bierman PJ. Current and emerging pharmacotherapies for primary CNS lymphoma. Clin Med Insights Oncol 2012; 6:219-231

11 Prica A, Chan K, Cheung MC. Combined modality therapy versus chemotherapy alone as an induction regimen for primary central nervous system lymphoma: a decision analysis. $\mathrm{Br} \mathrm{J}$ Haematol 2012;158(5):600-607

12 Thiel E, Korfel A, Hinkelbein W. Primary CNS lymphoma: chemotherapy followed by radiotherapy or chemotherapy alone? A randomized multicentric study. Front Radiat Ther Oncol 1999;33:349-353

13 Freilich RJ, Delattre JY, Monjour A, DeAngelis LM. Chemotherapy without radiation therapy as initial treatment for primary CNS lymphoma in older patients. Neurology 1996;46(2):435-439

14 Citterio G, Ferreri AJ, Reni M. Current uses of radiation therapy in patients with primary CNS lymphoma. Expert Rev Anticancer Ther 2013;13(11):1327-1337

15 Chamberlain MC. Treatment of primary CNS lymphoma (PCNSL) following successful treatment of systemic non-Hodgkin's lymphoma (NHL): a case series. J Neurooncol 2013;113(1):27-32

16 Rubenstein JL, Gupta NK, Mannis GN, Lamarre AK, Treseler P. How I treat CNS lymphomas. Blood 2013;122(14):2318-2330

17 Weller M, Martus P, Roth P, Thiel E, Korfel A; German PCNSL Study Group. Surgery for primary CNS lymphoma? Challenging a paradigm. Neuro-oncol 2012;14(12):1481-1484

18 Hiraga S, Arita N, Ohnishi T, et al. Rapid infusion of high-dose methotrexate resulting in enhanced penetration into cerebrospinal fluid and intensified tumor response in primary central nervous system lymphomas. J Neurosurg 1999;91(2):221-230

19 Joerger M, Huitema AD, Krähenbühl S, et al. Methotrexate area under the curve is an important outcome predictor in patients with primary CNS lymphoma: a pharmacokinetic-pharmacodynamic analysis from the IELSG no. 20 trial. Br J Cancer 2010;102(4):673-677

20 Neuwelt EA, Goldman DL, Dahlborg SA, et al. Primary CNS lymphoma treated with osmotic blood-brain barrier disruption: prolonged survival and preservation of cognitive function. J Clin Oncol 1991;9(9):1580-1590

21 Angelov L, Doolittle ND, Kraemer DF, et al. Blood-brain barrier disruption and intra-arterial methotrexate-based therapy for newly diagnosed primary CNS lymphoma: a multi-institutional experience. J Clin Oncol 2009;27(21):3503-3509

22 Khan RB, Shi W, Thaler HT, DeAngelis LM, Abrey LE. Is intrathecal methotrexate necessary in the treatment of primary CNS lymphoma? J Neurooncol 2002;58(2):175-178

23 Ferreri AJ, Reni M, Pasini F, et al. A multicenter study of treatment of primary CNS lymphoma. Neurology 2002;58(10):1513-1520

24 Boehme V, Zeynalova S, Kloess M, et al; German High-Grade NonHodgkin's Lymphoma Study Group (DSHNHL). Incidence and risk factors of central nervous system recurrence in aggressive lymphoma-a survey of 1693 patients treated in protocols of the German High-Grade Non-Hodgkin's Lymphoma Study Group (DSHNHL). Ann Oncol 2007;18(1):149-157

25 Bashir RM, Bierman PJ, Vose JM, Weisenburger DD, Armitage JO. Central nervous system involvement in patients with diffuse aggressive non-Hodgkin's lymphoma. Am J Clin Oncol 1991; 14(6):478-482

26 Tomita N, Kodama F, Sakai R, et al. Predictive factors for central nervous system involvement in non-Hodgkin's lymphoma: significance of very high serum LDH concentrations. Leuk Lymphoma 2000;38(3-4):335-343

27 Wolf MM, Olver IN, Ding JC, Cooper IA, Liew KH, Madigan JP. NonHodgkin's lymphoma involving the central nervous system. Aust $\mathrm{N}$ Z J Med 1985;15(1):16-21

28 van Besien K, Ha CS, Murphy S, et al. Risk factors, treatment, and outcome of central nervous system recurrence in adults with intermediate-grade and immunoblastic lymphoma. Blood 1998; 91(4):1178-1184

29 Zinzani PL, Magagnoli M, Frezza G, et al. Isolated central nervous system relapse in aggressive non-Hodgkin's lymphoma: the Bologna experience. Leuk Lymphoma 1999;32(5-6):571-576

30 Kridel R, Dietrich PY. Prevention of CNS relapse in diffuse large Bcell lymphoma. Lancet Oncol 2011;12(13):1258-1266

31 Roth P, Martus P, Kiewe P, et al. Outcome of elderly patients with primary CNS lymphoma in the G-PCNSL-SG-1 trial. Neurology 2012;79(9):890-896

32 Fritsch K, Kasenda B, Hader C, et al. Immunochemotherapy with rituximab, methotrexate, procarbazine, and lomustine for primary CNS lymphoma (PCNSL) in the elderly. Ann Oncol 2011;22(9): 2080-2085

33 Illerhaus G, Marks R, Müller F, et al. High-dose methotrexate combined with procarbazine and CCNU for primary CNS lymphoma in the elderly: results of a prospective pilot and phase II study. Ann Oncol 2009;20(2):319-325

34 Kasenda B, Schorb E, Fritsch K, Finke J, Illerhaus G. Prognosis after high-dose chemotherapy followed by autologous stem-cell transplantation as first-line treatment in primary CNS lymphoma-a long-term follow-up study. Ann Oncol 2012;23(10): 2670-2675

35 Kurzwelly D, Glas M, Roth P, et al. Primary CNS lymphoma in the elderly: temozolomide therapy and MGMT status. J Neurooncol 2010;97(3):389-392

36 Colocci N, Glantz M, Recht L. Prevention and treatment of central nervous system involvement by non-Hodgkin's lymphoma: a review of the literature. Semin Neurol 2004;24(4):395-404

37 Hegde U, Filie A, Little RF, et al. High incidence of occult leptomeningeal disease detected by flow cytometry in newly diagnosed aggressive B-cell lymphomas at risk for central nervous system involvement: the role of flow cytometry versus cytology. Blood 2005;105(2):496-502

38 Spectre G, Gural A, Amir G, Lossos A, Siegal T, Paltiel O. Central nervous system involvement in indolent lymphomas. Ann Oncol 2005;16(3):450-454

39 MacKintosh FR, Colby TV, Podolsky WJ, et al. Central nervous system involvement in non-Hodgkin's lymphoma: an analysis of 105 cases. Cancer 1982;49(3):586-595

40 Gleissner B, Siehl J, Korfel A, Reinhardt R, Thiel E. CSF evaluation in primary CNS lymphoma patients by PCR of the CDR III IgH genes. Neurology 2002;58(3):390-396

41 Go JL, Lee SC, Kim PE. Imaging of primary central nervous system lymphoma. Neurosurg Focus 2006;21(5):E4

42 Haldorsen IS, Espeland A, Larsson EM. Central nervous system lymphoma: characteristic findings on traditional and advanced imaging. AJNR Am J Neuroradiol 2011;32(6):984-992

43 Boehme V, Schmitz N, Zeynalova S, Loeffler M, Pfreundschuh M. CNS events in elderly patients with aggressive lymphoma treated with modern chemotherapy (CHOP-14) with or without rituximab: an analysis of patients treated in the RICOVER-60 trial of the German High-Grade Non-Hodgkin Lymphoma Study Group (DSHNHL). Blood 2009;113(17):3896-3902

$44 \mathrm{Kim}$ SJ, Oh SY, Kim JS, et al. Secondary central nervous system (CNS) involvement in patients with diffuse large B-cell lymphoma: a therapeutic dilemma. Ann Hematol 2011;90(5):539-546 